\title{
Erratum to: A set of invariant quality factors measuring the deviation from the Kerr metric
}

\author{
Alfonso García-Parrado Gómez-Lobo • \\ José M. M. Senovilla
}

\section{Erratum to: Gen Relativ Gravit DOI 10.1007/s10714-013-1518-9}

The original version of this article unfortunately contained mistakes. Two equation numbers were not displayed:

- In the line below Eq. (36), the number should be (10)

- In the line above Eq. (42), the number should be (10)

The online version of the original article can be found under doi:10.1007/s10714-013-1518-9.

A. G.-P. Gómez-Lobo ( $\varangle)$

Centro de Matemática, Universidade do Minho, 4710-057 Braga, Portugal

e-mail: alfonso@math.uminho.pt

J. M. M. Senovilla

Física Teórica, Universidad del País Vasco, Apartado 644, 48080 Bilbao, Spain

e-mail: josemm.senovilla@ehu.es 